\title{
City Information Modeling como ferramenta na gestão urbana: aplicação da integração GIS-BIM
}

\author{
City Information Modeling as a tool in urban management: application of GIS-BIM integration \\ El modelado de información urbana como herramienta de gestión urbana: aplicación de la
} integración GIS-BIM

Recebido: 19/01/2022 | Revisado: 23/01/2022 | Aceito: 31/01/2022 | Publicado: 01/02/2022

Francisca Ires Vieira de Melo

ORCID: https://orcid.org/0000-0002-2537-0089 Universidade Federal do Rio Grande do Norte, Brasil E-mail: ires_vieira@hotmail.com

Josyanne Pinto Giesta ORCID: https://orcid.org/0000-0002-0596-3821 Instituto Federal de Educação, Ciência e Tecnologia do Rio Grande do Norte, Brasil E-mail: josyanne.giesta@ifrn.edu.br

Luiz Alessandro Pinheiro da Camara de Queiroz ORCID: https://orcid.org/0000-0002-4240-8384 Universidade Federal do Rio Grande do Norte, Brasil E-mail: alessandro.camara@ufrn.br

\begin{abstract}
Resumo
Os centros urbanos vêm se tornando ambientes cada vez mais complexos, ocasionando mudanças que se expressam tanto na paisagem da cidade como no comportamento e estilo de vida das pessoas, causando, dessa forma, transformações que impactam diretamente na estruturação socioespacial urbana. Deste modo, esse artigo apresenta parte dos resultados de uma pesquisa de mestrado, possuindo o objetivo de elaborar diretrizes para a integração de softwares GIS-BIM, com intuito de facilitar a tomada de decisões no planejamento urbano. Para isso, será abordado o método de pesquisa Design Science Research (DSR) com a utilização de artefatos destinados a sanar problemas do mundo real. Apresenta-se abordagens de integração GIS-BIM, por meio da compatibilização do georreferenciamento e da modelagem de um bairro da cidade de Pau dos Ferros/RN - Brasil. Como resultados desenvolveu-se uma proposta com diretrizes de integração GIS-BIM e por meio da aplicação da mesma foi possível obter a modelagem total do bairro com 1.496 edificações modeladas, onde os parâmetros de gabarito, área construída e área de projeção da edificação podem ser extraídos de forma automática. O desenvolvimento dessa integração contribui na redução do custo de tempo e de recursos para produzir informações geográficas, além de facilitar a visualização e compreensão do território estudado.
\end{abstract}

Palavras-chave: Planejamento urbano; Modelagem em massa; CIM.

\begin{abstract}
Urban centers are becoming increasingly complex environments, causing changes that are expressed both in the city landscape and in people's behavior and lifestyle, thus causing transformations that directly impact the urban sociospatial structuring. In this way, this paper presents part of the results of a master's research, with the objective of developing guidelines for the integration of GIS-BIM software, in order to facilitate decision making in urban planning. To this end, the Design Science Research (DSR) method will be used, using artifacts to solve real-world problems. It presents approaches for GIS-BIM integration, through the compatibility of georeferencing and modeling of a neighborhood in the city of Pau dos Ferros/RN - Brazil. As results, a proposal with guidelines for GIS-BIM integration was developed, and by applying it, it was possible to obtain the total modeling of the neighborhood with 1,496 modeled buildings, where the parameters of gauge, built area and projection area of the building can be extracted automatically. The development of this integration contributes to reducing the cost of time and resources to produce geographic information, as well as facilitating the visualization and understanding of the territory studied.
\end{abstract}

Keywords: Urban planning; Mass modeling; CIM.

\section{Resumen}

Los centros urbanos se están convirtiendo en entornos cada vez más complejos, lo que provoca cambios que se expresan tanto en el paisaje de la ciudad como en el comportamiento y el estilo de vida de las personas, provocando así transformaciones que afectan directamente a la estructuración socioespacial urbana. De este modo, este trabajo presenta parte de los resultados de una investigación de máster, con el objetivo de desarrollar directrices para la 
integración de software GIS-BIM, con el fin de facilitar la toma de decisiones en la planificación urbana. Para ello, se abordará el método de investigación en ciencias del diseño (DSR) con el uso de artefactos destinados a resolver problemas del mundo real. Se presentan enfoques de integración SIG-BIM, a través de la compatibilidad de la georreferenciación y el modelado de un barrio de la ciudad de Pau dos Ferros/RN - Brasil. Como resultados se desarrolló una propuesta con directrices para la integración GIS-BIM y a través de la aplicación de la misma se pudo obtener el modelado total del barrio con 1.496 edificios modelados, donde los parámetros de gálibo, área construida y área de proyección del edificio pueden ser extraídos automáticamente. El desarrollo de esta integración contribuye a reducir el coste de tiempo y recursos para producir información geográfica, además de facilitar la visualización y comprensión del territorio estudiado.

Palabras clave: Planificación urbana; Modelización de masas; CIM.

\section{Introdução}

O relevante processo migratório de pessoas para os centros urbanos, advindo desde a revolução industrial, ocasionou um aumento da concentração populacional nas zonas urbanas, esse movimento faz parte de um conjunto de mudanças que irão se expressar, tanto na paisagem urbana da cidade, como no comportamento e estilo de vida das pessoas, causando transformações que irão impactar diretamente na estruturação da dinâmica socioespacial das cidades. Diante dessa problemática o termo urbanização se expressa como um processo de transformação de uma determinada região, representando não somente o crescimento da população das cidades, mas também um conjunto de técnicas e de obras que permitem dotar uma cidade ou área da cidade de condições de infraestrutura, planejamento e organização administrativa (Monteiro \& Veras, 2017).

Almeida e Andrade (2018) abordam a cidade como território físico no qual agem dinâmicas expansivas, que respondem de maneira própria no surgimento de novos desafios, tais como redes de mobilidade saturadas, alagamentos, crises de fornecimento de energia e água, manejo ineficiente de resíduos sólidos, decadência das redes físicas urbanas, desequilíbrios microclimáticos, catástrofes naturais, entre outros. Diante desses fatores, manifestou-se a necessidade de investigar meios de planejar e compreender o crescimento das cidades, de modo a considerar os seus aspectos sociais, políticos, econômicos e ideológicos, que envolvem este processo ao longo do tempo, iniciando assim, a busca por integrar essas condições com as facilidades que o meio tecnológico pode ofertar, surgindo desse modo os estudos em relação ao termo City Information Modeling (CIM).

Nesse contexto, surgiu com Khemlani (2005), os primeiros termos sobre o conceito CIM, abordando o tema como uma extensão do conceito Building Information Modeling (BIM) para cidades, criando uma possível utilização que auxilie na elaboração de uma réplica digital e precisa de uma cidade, e assim ser submetida a simulações de impactos de decisões e análises holísticas.

Diante do exposto, com o elevado número de características propiciadas pelas ferramentas digitais, elas se tornam grandes aliadas nesse processo, evidenciando seu potencial para mudar a forma de conceber, planejar, projetar, construir e operar as cidades, tornando-as mais adequadas possíveis para os seus habitantes. Assim, o termo City Information Modeling emerge atrelado a questão da interoperabilidade entre as tecnologias Building Information Modeling (BIM) e Geographic Information System (GIS), encontrando como uma das mais relevantes dificuldades o formato dos arquivos entre o GIS e o BIM, que dificulta o estabelecimento de interações de informação entre a escala do edifício e a escala da cidade (Deritti, 2018).

É importante destacar as contribuições que as ferramentas GIS e BIM podem favorecer na etapa de modelagem espacial e territorial. O GIS é uma ferramenta voltada para o geoprocessamento de informações e pode auxiliar na visualização de formas territoriais em grande escala, por meio dele é possível criar mapas temáticos, dependendo apenas de informações base. O BIM é uma ferramenta de modelagem tridimensional da informação, com o foco mais voltado para construções, onde por meio da mesma é possível unir todas as informações referentes a cada etapa de uma determinada obra/projeto. 
Embora a aplicação da modelagem 3D para cidades já tenha sido bem abordada em estudos anteriores, como Reitz e Banz (2014), Arrubla e Chavez (2016), Osipov e Chekodaev (2019), ainda é limitada a atenção dos estudos voltados ao eixo de integração GIS-BIM como facilitador na gestão das cidades, uma vez que grande parte das pesquisas apresentadas se concentram apenas na realização da modelagem 3D ou mapeamento urbano, sem ter uma integração de informações a essa modelagem, e em como pode ser feita a disponibilidade desse produto final como ferramenta útil para gestores.

Assim, sabendo-se dos desafios que surgem na gestão das cidades e das demandas em diferentes áreas, como na segurança pública, saúde, defesa civil, construção civil, mobilidade urbana, entre outros, o presente estudo busca propor diretrizes de integração GIS-BIM e analisar sua aplicação, por meio da utilização de dados GIS como ponto base para elaboração da modelagem 3D, de um bairro da cidade de Pau dos Ferros/RN, de modo que ela possa ser utilizada como suporte na tomada de decisão da gestão urbana.

\section{Aspectos Metodológicos}

Esta pesquisa visa propor diretrizes de modelagem integrando GIS-BIM e procedimentos de análise de parâmetros na aplicação do CIM no auxílio a tomada de decisões na gestão urbana. Com isso, optou-se pelo método de pesquisa Design Science Research (DSR) para o desenvolvimento deste estudo. A DSR é um método de pesquisa que lida com dois tipos de problemas, os problemas práticos, que demandam de uma mudança no mundo que melhor concorde com os objetivos dos tomadores de decisão relacionados ao problema, e os problemas de conhecimento, que demandam uma mudança em nosso conhecimento sobre o mundo (Wieringa, 2009). Para Dresch et al., (2015) a DSR busca a partir da compreensão do problema, transformar as situações através do desenvolvimento e análise de artefatos que permitam alterar as condições atuais para cenários melhores ou desejáveis.

Esse método se baseia na utilização de artefatos destinados a sanar problemas do mundo real, possibilitando o desenvolvimento de uma teoria consistente no contexto da pesquisa (Lukka, 2003). March e Smith (1995) classificaram os artefatos em quatro categorias que são elas: constructos, modelos, métodos e instâncias. Diante do exposto, na presente pesquisa, conforme a classificação de March e Smith (1995) será elaborado um método, o qual abordara uma sequência de passos que auxiliam para a realização de determinada atividade.

\subsection{Etapas da Pesquisa}

Conforme abordado por Dresch et al., (2015) vários autores elaboraram diferentes métodos para a condução das pesquisas fundamentadas na DSR, dos quais as similaridades entre eles podem ser identificadas nas seguintes fases de delineamento da pesquisa: conscientização do problema, sugestão, desenvolvimento do artefato, avaliação e conclusão. Diante disso, a presente pesquisa será condicionada as 5 etapas citadas acima, (Figura 1). 
Figura 1: Atividades da pesquisa.

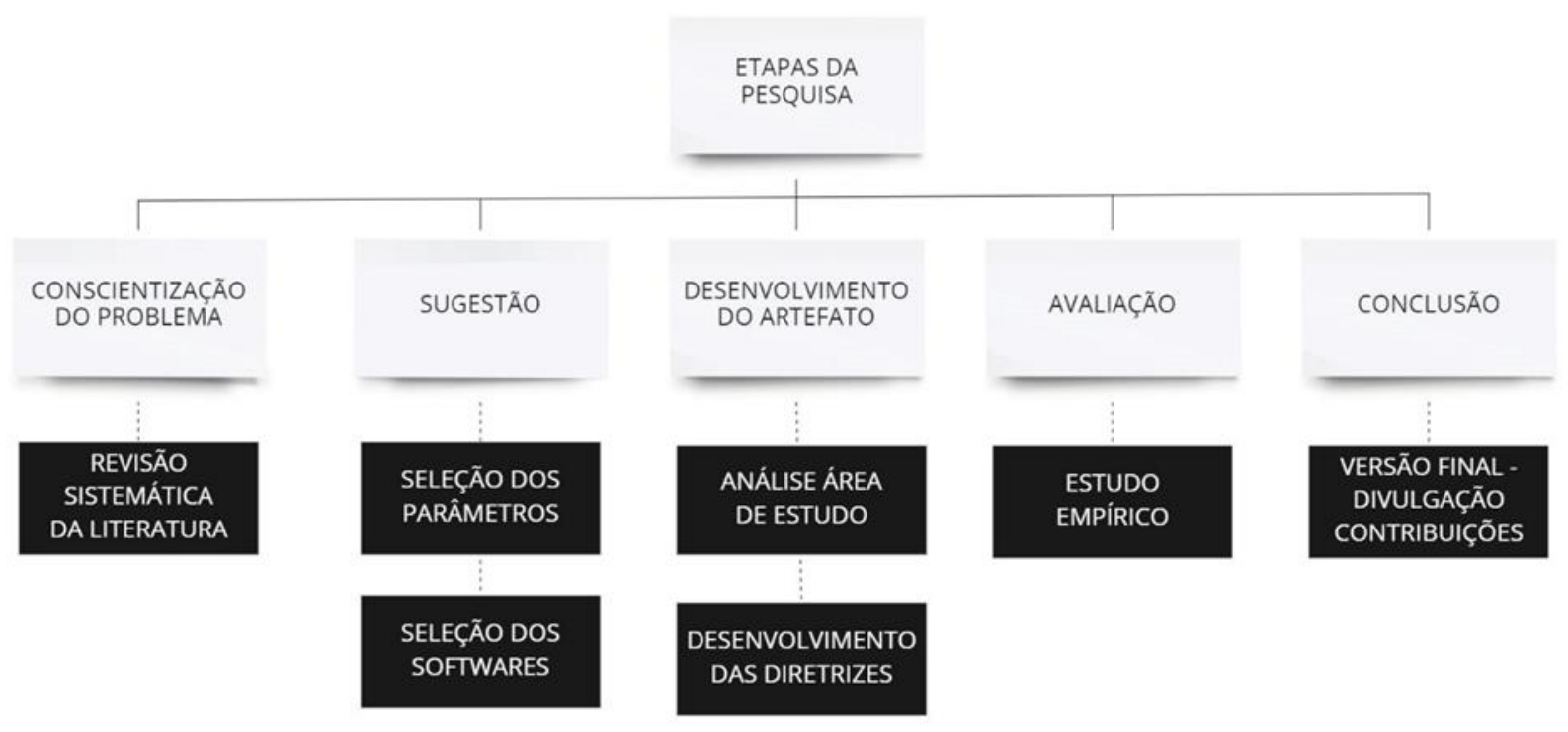

Fonte: Autores (2021).

\subsubsection{Etapa 1: Conscientização do problema}

A etapa inicial, se fundamenta na formulação e conscientização do problema a ser estudado, com base na revisão sistemática da literatura (RSL), que consiste no método sistemático, abrangente e reprodutível para identificar, avaliar e sintetizar o corpo existente de trabalhos completos e registrados, produzidos por pesquisadores, estudiosos e profissionais (Fink, 2005). Almejou-se por meio da RSL identificar e caracterizar os estudos existentes na literatura sobre a implementação e aplicação do CIM, com a integração GIS-BIM, assim como na identificação dos principais softwares aplicados nesta temática. Na mesma realizou-se ao final da aplicação dos critérios de inclusão e exclusão, o estudo de 64 artigos para a amostra da RSL, as etapas seguidas são apresentadas na Figura 2.

Figura 2: Etapas seguidas na realização da RSL.

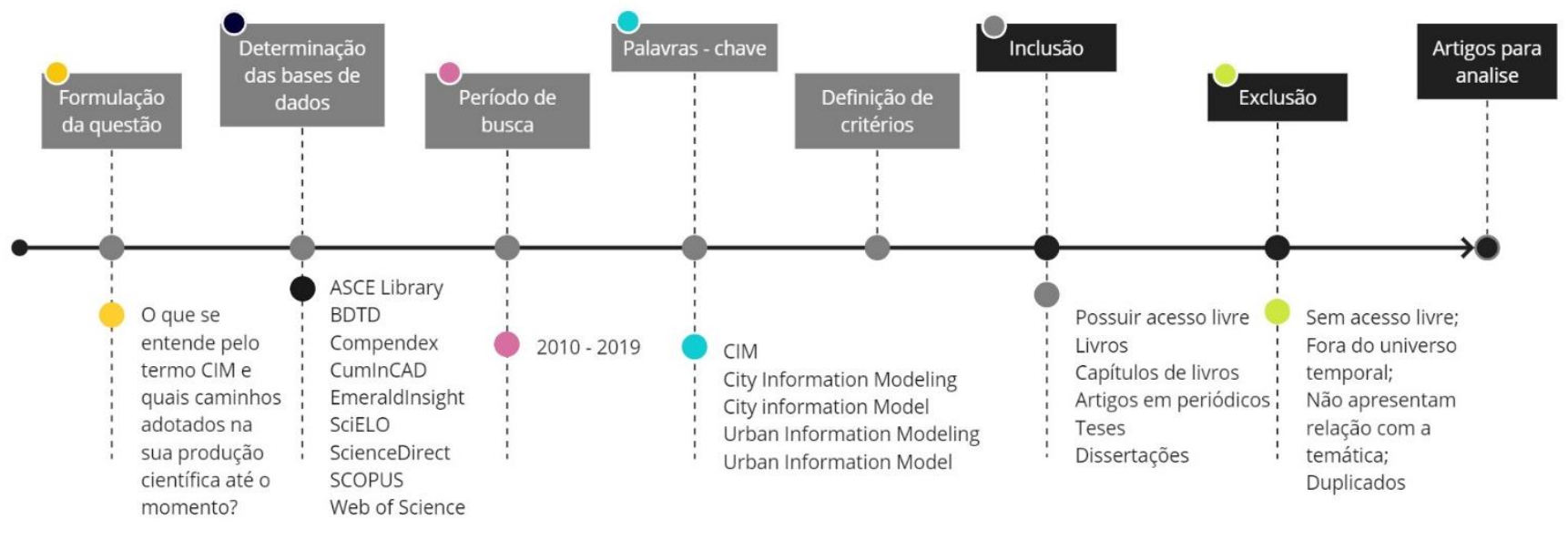

Fonte: Autores (2021). 


\subsubsection{Etapa 2: Sugestão}

A etapa de sugestão é o momento no qual o pesquisador propõe ideias e concepções que norteiam o desenvolvimento do estudo. Conforme Reitz e Banz (2014) gerenciar os ambientes urbanos em 3D está se tornando cada vez mais importante e complexo, neste sentido sugerem como solução a utilização de softwares para criar, analisar e manter uma cidade 3D. Por tanto, será proposto diretrizes de integração GIS-BIM na modelagem urbana para auxiliar na análise de parâmetros perante o uso e ocupação do solo urbano, com o intuito de facilitar as tomadas de decisões por parte dos planejadores urbanos.

\subsubsection{Estudo dos parâmetros}

No auxílio a gestão e controle urbanístico, especialmente quando se relaciona a intensificação do uso e ocupação do solo à capacidade de infraestrutura, torna-se imprescindível, a análise de parâmetros urbanísticos de uso e ocupação do solo urbano (Dantas, 2013). Os espaços urbanos são diretamente influenciados pelos instrumentos do planejamento urbano, desde a sua forma de uso até sua ocupação. Dentre esses instrumentos tem-se, por exemplo, o Plano Diretor, que se dá como um conjunto de regras, constituído em forma de documento orientador, apresentando à aplicação dos recursos regulamentados, dentre eles os parâmetros urbanísticos que servem como ferramenta para regular a densidade urbana e sua volumetria (Instituto Polis, 2002).

De acordo com Barbosa (2013) as definições e alterações morfológicas influenciam diretamente a qualidade dos espaços, a mobilidade e o conforto bioclimático local. Por meio da análise dos parâmetros urbanísticos que estão relacionados também com características físicas da área, como suas conformações geométricas, geometrias das edificações, é possível buscar melhorias no desempenho ambiental e social dos espaços urbanos.

Os parâmetros urbanísticos presentes, geralmente, no instrumento do Plano Diretor se referem ao gabarito de altura máxima permitida para as edificações, aos afastamentos e recuos, à área construída, à taxa de ocupação, ao coeficiente de aproveitamento e à taxa de permeabilidade. Com isso, os parâmetros que serão analisados por meio da modelagem do bairro serão: taxa de ocupação, e número máximo de pavimentos, e área construída tendo como critério de escolha além de serem alguns dos parâmetros mais importantes na análise do uso e ocupação do solo, o fato dos mesmos não necessitarem de uma modelagem muito rica em informações e detalhes.

\subsubsection{Estudo dos softwares}

Esta fase envolve a seleção dos softwares a serem utilizados no desenvolvimento do artefato. Deste modo, definiu-se por utilizar o software livre e de código aberto Qgis que é um projeto oficial da Open Source Geospatial Foundation (OSGeo), na etapa de manipulação de mapas e georreferenciamento, a escolha se deu pelo mesmo ser um software livre, e por permitir exportar dados compatíveis com o software utilizado para modelagem 3D. Para etapa de modelagem optou-se pelo CityEngine, que é um software de modelagem urbana da empresa Esri, o mesmo se destaca dentre as outras opções pelo fato de permitir a criação de ambientes urbanos imersivos e interativos, podendo importar arquivos de softwares GIS para recortar e importar imagens de satélite e terreno 3D, e também dados de pegadas de ruas e edifícios do OpenStreetMap. Além de permitir a exportação da modelagem 3D finalizada para a web e experiências de Realidade Virtual, de modo que possa facilitar o acesso de gestores e planejadores sem a necessidade de ter o software diretamente instalado.

\subsubsection{Etapa 3: Desenvolvimento}

Esta etapa compreende a elaboração do artefato, para isso foram desenvolvidas as subetapas descritas nos itens a seguir. 


\subsubsection{Análise da área de estudo}

Para aplicação do presente estudo foi selecionada a cidade de Pau dos Ferros/RN, visto que ela se encontra com um desenvolvimento contínuo e acelerado, acarretado por suas características de polo assistencial para as demais cidades vizinhas, aumentando ainda mais sua perspectiva de desenvolvimento, sem possuir um planejamento urbano bem estruturado. Em um contexto histórico, a cidade apresenta fundação datada do ano de 1856 e está situada na região Alto Oeste Potiguar do estado do Rio Grande do Norte, a 392 km da capital Natal, com área territorial de 259,959 km², e população estimada de 30.000 habitantes, sua localização pode ser observada na Figura 3 (IBGE; 2012).

Figura 3: Localização de Pau dos Ferros no RN no mapa do Brasil.

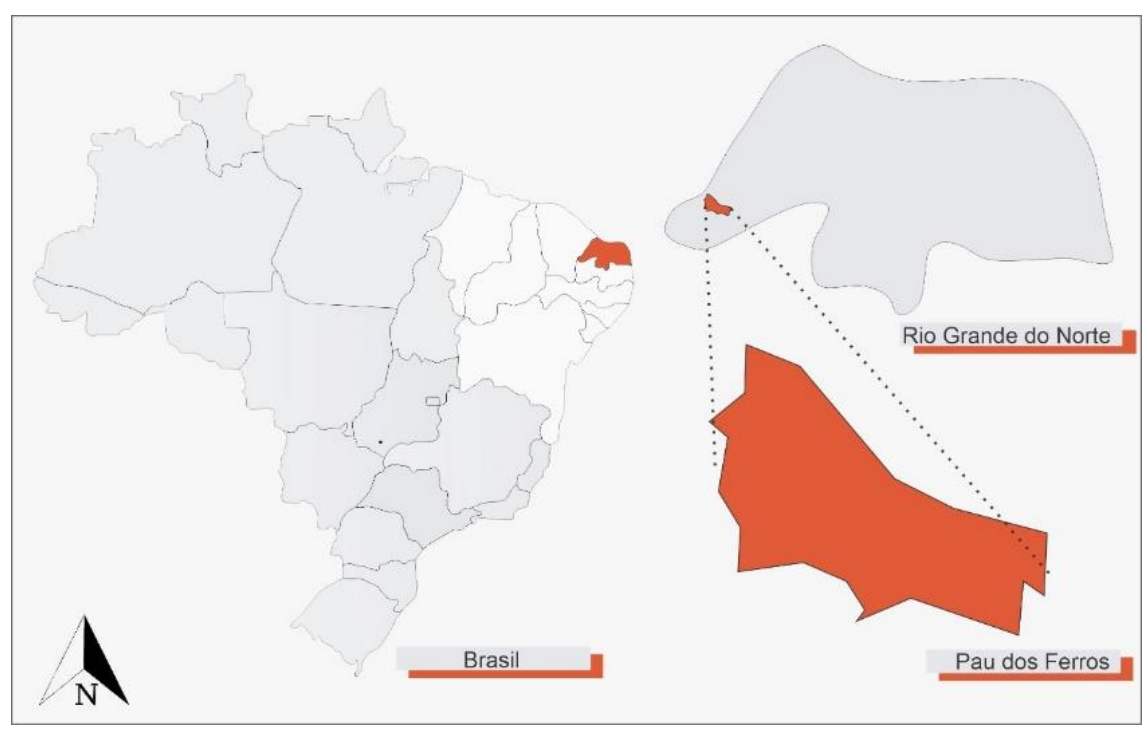

Fonte: Adaptado de IBGE (2021).

A cidade de Pau dos Ferros é zoneada em 24 bairros, conforme Figura 4. Para essa pesquisa, selecionou-se o bairro São Judas Tadeu, para aplicação da modelagem e posterior análise dos seus parâmetros. A escolha se deu por critério geográfico, uma vez que sua localização é próxima ao centro da cidade, apresentando densidade e consolidação ideal para análise nesse estudo. 
Figura 4: Zoneamento dos bairros de Pau dos Ferros.

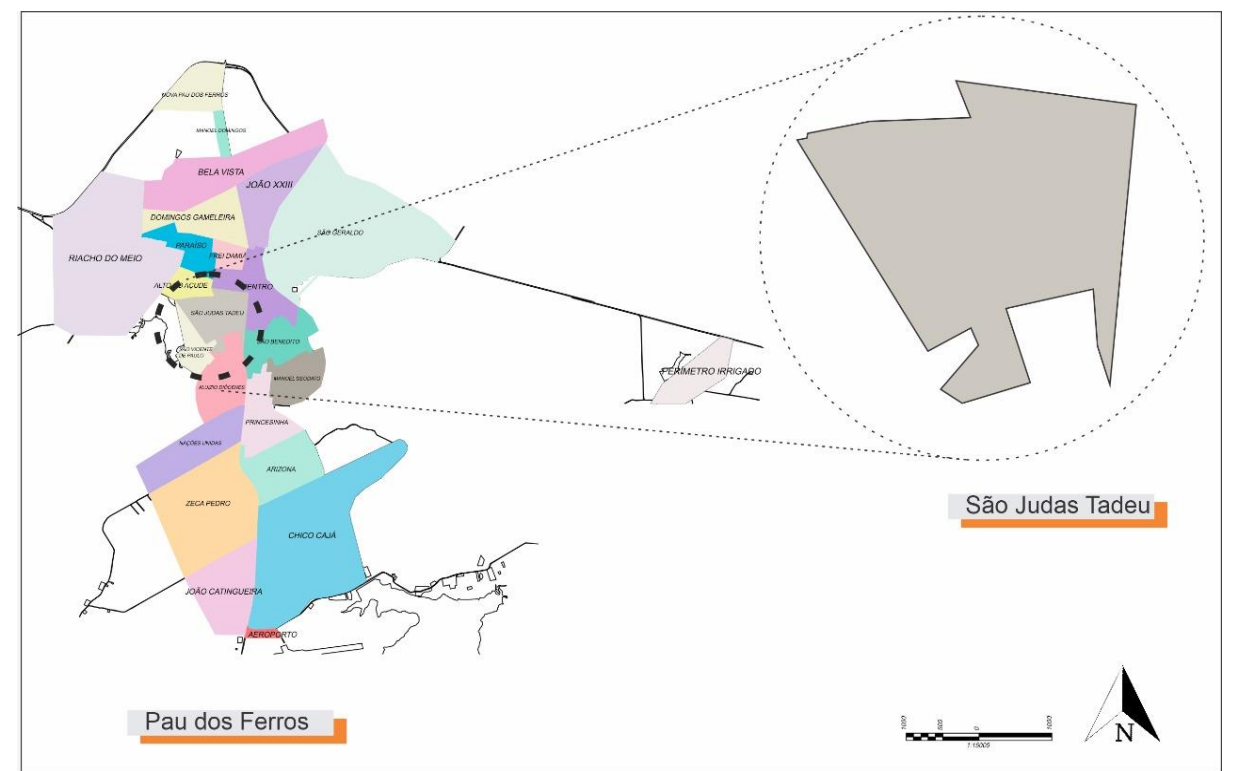

Fonte: Adaptado de Projeto Smart City, Núcleo de pesquisa e extensão Acesso à Terra Urbanizada (2020).

\subsubsection{Desenvolvimento diretrizes}

Nesta etapa foram desenvolvidas as diretrizes de integração GIS-BIM, desde a obtenção dos dados georreferenciados até a elaboração de uma visualização tridimensional desses dados, e aplicação das mesmas em um estudo de caso da modelagem do bairro, de forma a elaborar um método capaz de ser replicado em outros bairros e cidades.

\subsubsection{Etapa 4: Avaliação}

Etapa onde ocorreu a avaliação do artefato quanto a sua aplicabilidade por meio do estudo empírico, desenvolvendo a modelagem de um bairro com a aplicação das diretrizes elaboradas.

\subsubsection{Tratamento inicial dos dados}

Inicialmente foi realizado uma seleção e limpeza dos dados georreferenciados que foram cedidos pelo Projeto Smart City, Núcleo de pesquisa e extensão Acesso à Terra Urbanizada (2020), onde as shapefiles continham os dados referentes a alguns bairros, quadras, lotes e edificações, da cidade de Pau dos Ferros, Figura 5. 
Figura 5: Arquivos shapefiles antes da seleção da área de estudo.

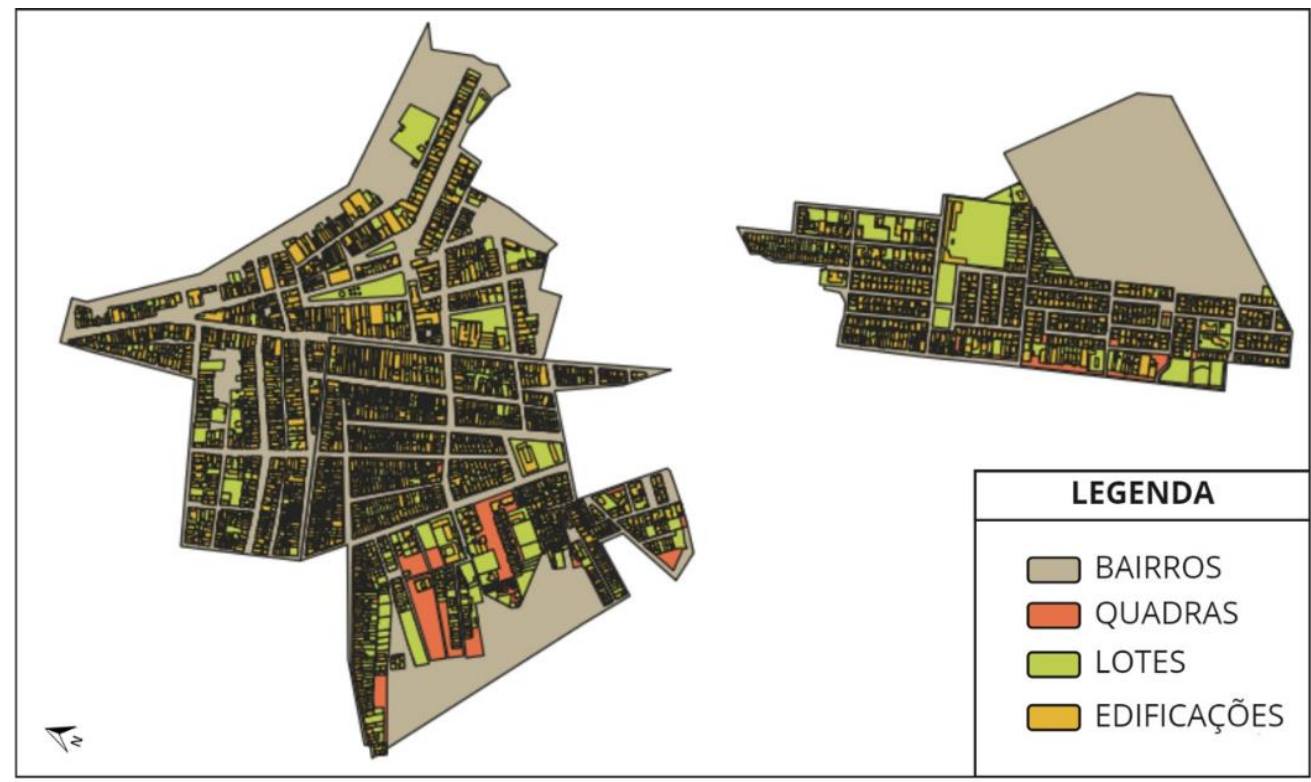

Fonte: Autores (2021).

Com isso, de modo a fazer uma seleção apenas dos dados referentes a área de estudo, o bairro São Judas Tadeu, foram realizadas algumas manipulações nesses arquivos, no software Qgis (versão 3.18.2), Figura 6.

Figura 6: Arquivos shapefiles após a seleção da área de estudo.

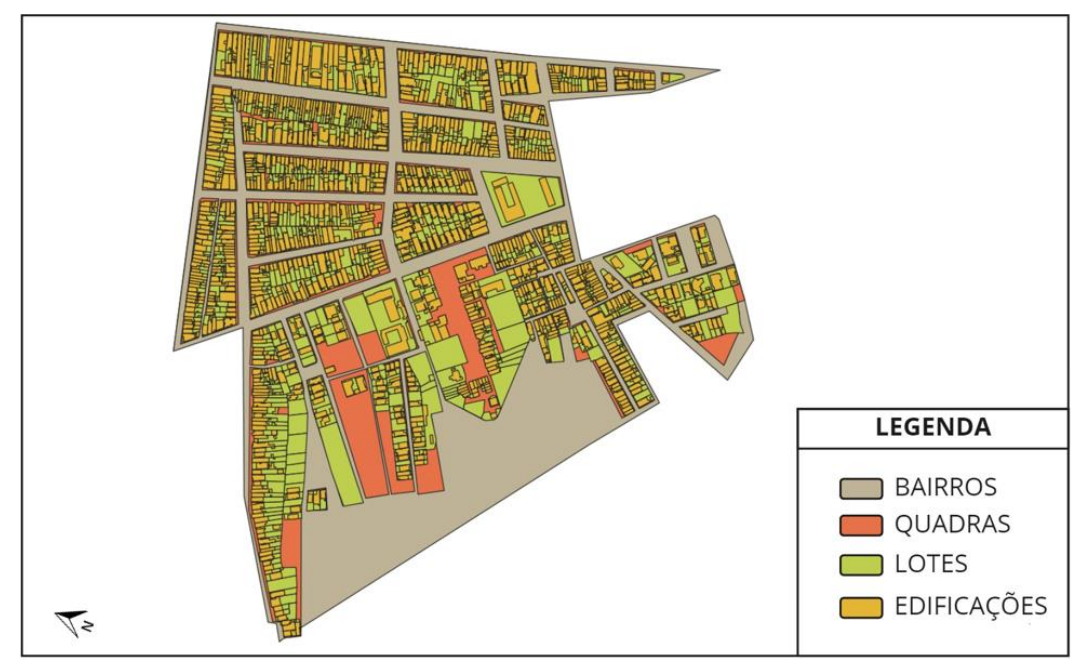

Fonte: Autores (2021)

\subsubsection{Categorização das edificações}

Nesta etapa os dados foram importados para o software CityEngine (versão 2019.0) e sincronizados com o mapa da área de estudo, Figura 7. Após isso, iniciou-se a categorização das edificações, onde foram conferidas cada rua do bairro, edificação por edificação, observando o número de pavimentos que cada uma possuía. 
Research, Society and Development, v. 11, n. 2, e46711225930, 2022

(CC BY 4.0) | ISSN 2525-3409 | DOI: http://dx.doi.org/10.33448/rsd-v11i2.25930

Figura 7: Integração shapefile com mapa.

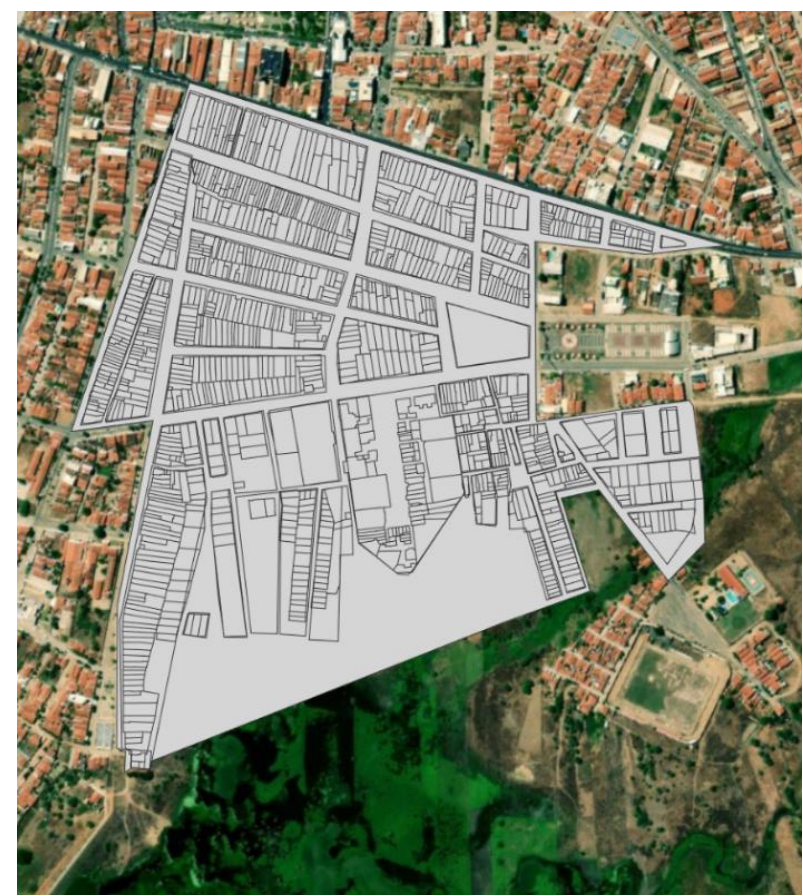

Fonte: Autores (2021)

Neste momento da pesquisa utilizou-se o Google Earth e visitas in loco entre o mês de junho e julho de 2021, para conferência das edificações. Simultaneamente no software CityEngine foram aplicadas texturas referentes a quantidade de pavimentos, sendo Branca para edificações Térrea (1 pavimento), Marrom (2 pavimentos), Verde (3 pavimentos) e Amarela (4 pavimentos), conforme Figura 8.

Figura 8: Aplicação das texturas.

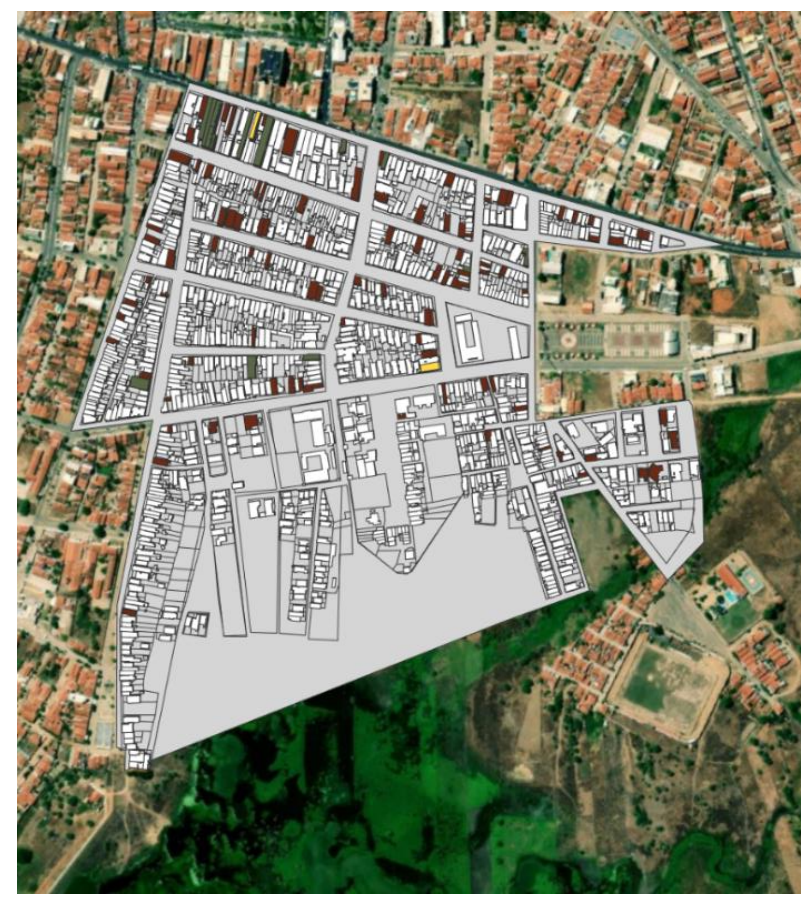

Fonte: Autores (2021). 


\subsubsection{Elaboração do algoritmo}

No software de modelagem utilizado (CityEngine) a geração de modelos pode ser feita por meio de código de regras ou python script. Assim como é possível criar modelo tridimensional de cidades, tendo como base a existência de dados topográficos. Deste modo, devido a finalidade da pesquisa ser direcionada a parametrização e extração de informações da modelagem, utilizou-se o módulo CGA, que é uma metodologia processual, na qual se utiliza a linguagem de programação chamada CGA (Computer Generated Architecture), que permite que as regras e indicadores urbanísticos sejam alterados, dando assim, liberdade e espaço para novas possibilidades de projetos e estudos urbanísticos.

As regras criadas nos ficheiros CGA são processadas, dentro do ambiente CityEngine. Elas são criadas por meio de programação no ambiente de desenvolvimento, e seguem as seguintes etapas:
i. Criação do ficheiro com nome da cena (por exemplo, scene.cej);
ii. Criação do ficheiro de regras, no caso o ficheiro CGA (Computer Generated Architecture);
iii. Elaboração do algoritmo com as regras no ficheiro CGA, recorrendo ao editor CGA;
iv. Determinação do ponto da regra inicial (@Start Rule);
v. Seleção das shape 2D que deseja aplicar as regras;
vi. Aplicação do ficheiro de regras nas shape 2D selecionadas;
vii. Execução das regras nos modelos, através do botão Generate.

Ao criar o conjunto de regras é possível fazer aplicação em grande escala, possibilitando a geração de modelos 3D em um curto período de tempo, na Figura 9 é apresentado o editor CGA, no qual é possível a criação e modificação do algoritmo com as regras.

Figura 9: Interface do editor CGA.

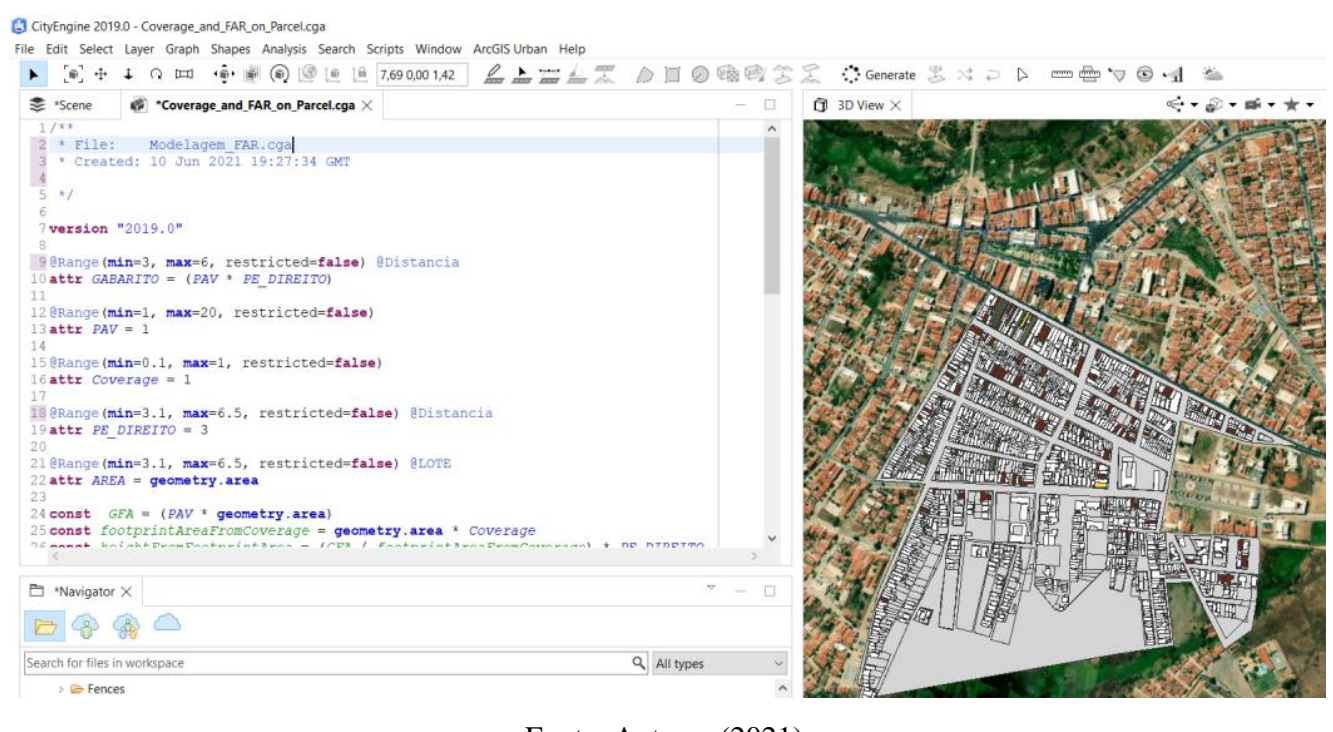

Fonte: Autores (2021).

O código de regras elaborado, de acordo com as necessidades da pesquisa é apresentado na Figura 10, ressaltando que não foi tomado como ponto relevante, nesta fase da pesquisa, o quesito de nível de detalhamento, ou seja, questão de 
aberturas de portas, janelas, desenho de telhados, entre outros. Este pode ser um ponto de regras acrescentado no algoritmo, para pesquisas futuras.

Levou-se em consideração, especialmente quando se relaciona a intensificação do uso e ocupação do solo e à capacidade de infraestrutura, desse modo as principais regras estão relacionadas com quantidade de pavimentos, altura do pé direito (altura de piso ao teto), gabarito e área construída e área de projeção da edificação.

Figura 10: Interface do algoritmo.

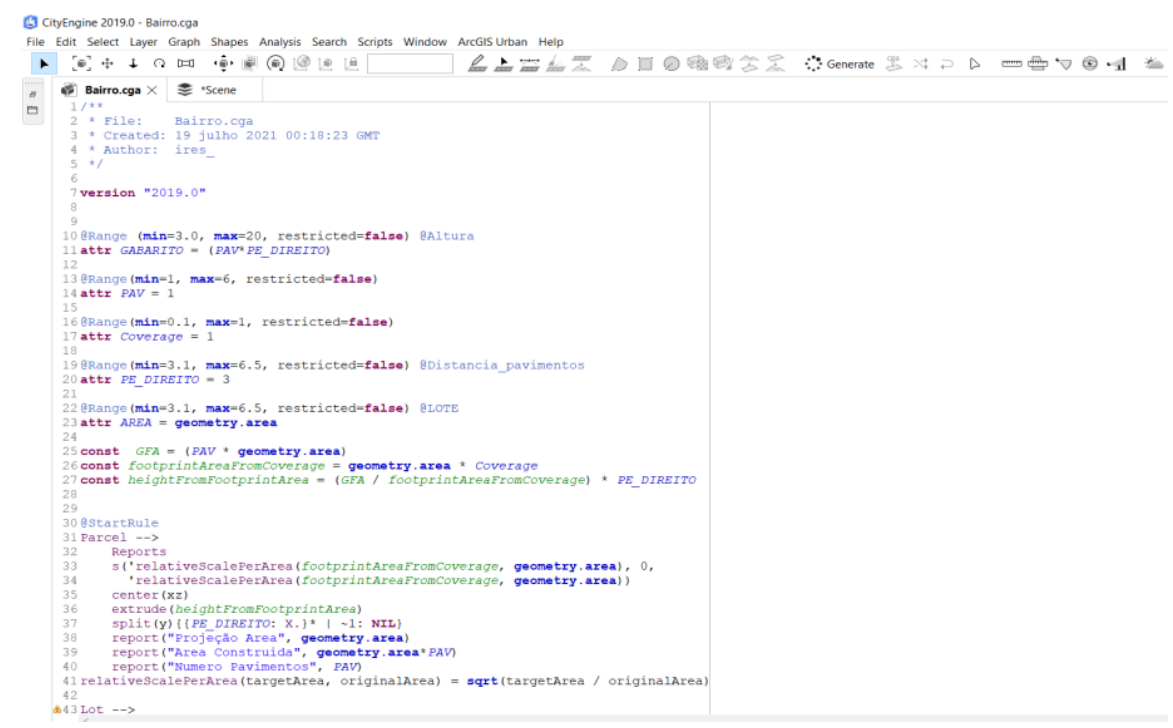

Fonte: Autores (2021).

\subsubsection{Etapa 5: Considerações finais}

Esta etapa de conclusão deve sintetizar o que se aprendeu em todas as fases do projeto, bem como justificar a contribuição do trabalho (Lacerda et al.; 2013).

\section{Resultados e Discussão}

De acordo com Liu et al. (2017), a integração entre o BIM e o GIS é fundamental para transformar informações em conhecimento e inteligência, impactando positivamente na solução de problemas nos setores, tanto da construção civil como da infraestrutura. Nos estudos que tratam sobre GIS-BIM, habitualmente o foco é mais direcionado, da parte do GIS, em buscar tecnologias de mapeamento como nos estudos de Costa Júnior e Cabral (2019), Costa, Reis Neto e Oliveira (2020) e Sousa, Vale, Barbosa, Silva e Sousa (2021) que utilizam as facilidades impostas pelo georreferenciamento para mapear zonas de estudo. E da parte do BIM, a capacidade de integrar informações a modelagem como em Arrubla e Chavez (2016) e Olszewski et al. (2016), que aplicam a modelagem na análise do processo de expansão urbana.

Diante do exposto, elaborou-se uma proposta com diretrizes de integração GIS-BIM, conforme Figura 11. A mesma baseia-se inicialmente na obtenção dos dados de georreferenciamento da área, elaborados em ferramentas GIS. Após isso realizou-se a importação dos arquivos Shapefile para o software CityEngine, onde os mesmos são integrados com o mapa real da área, posteriormente é realizada a aplicação dos tipos de texturas referentes ao mapeamento dos níveis de pavimentos das edificações. Em seguida foi desenvolvido o algoritmo de geração da modelagem 3D, visto ser aplicada a metodologia de modelagem processual, a qual é baseada na criação de um algoritmo com regras conforme o que se deseja obter de parâmetros. A partir da aplicação desse algoritmo, que ocorre dentro da própria plataforma do software CityEngine, obteve-se a geração 
automática da modelagem 3D das edificações, sendo manualmente ajustadas posteriormente os níveis dos pavimentos das edificações. Com a modelagem elaborada foi possível obter automaticamente parâmetros como área construída, área de projeção e gabarito da altura total das edificações.

Figura 11: Diretrizes de integração GIS-BIM.

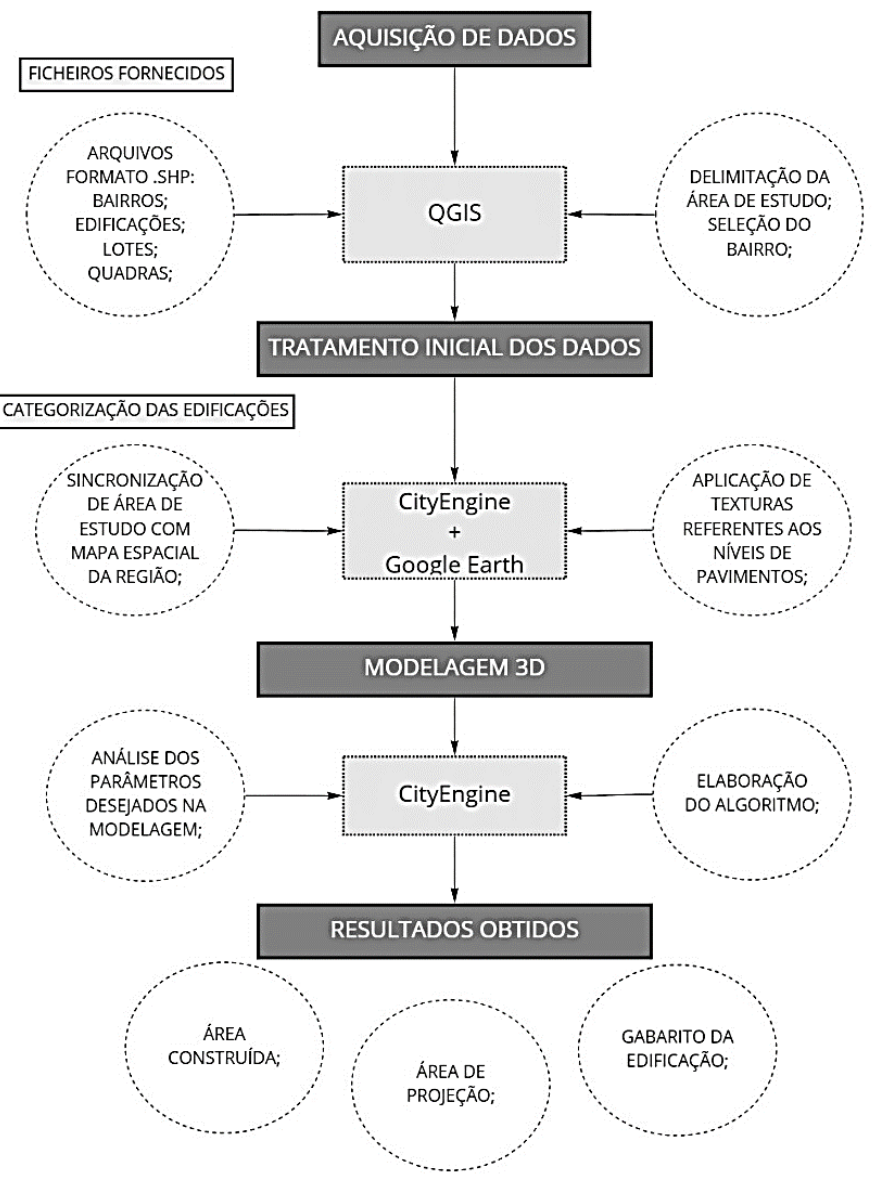

Fonte: Autores (2021)

Na primeira etapa de categorização das edificações, obteve-se a aplicação das texturas referentes aos pavimentos, em cada edificação do bairro, Figura 12. Com as regras definidas no algoritmo, quando aplicado na shape referente a edificação, ele gera um modelo 3D, de acordo com a geometria da área georreferenciada da shapefile, assim como permite que o usuário possa adicionar quantos pavimentos determinada edificação possuí, e qual o pé direito a ser adotado. Após a geração do modelo 3D, é possível observar os diferentes gabaritos das edificações, sendo atribuído um pé direto de 3 metros para todas as edificações. 
Figura 12: Categorização das edificações do bairro.

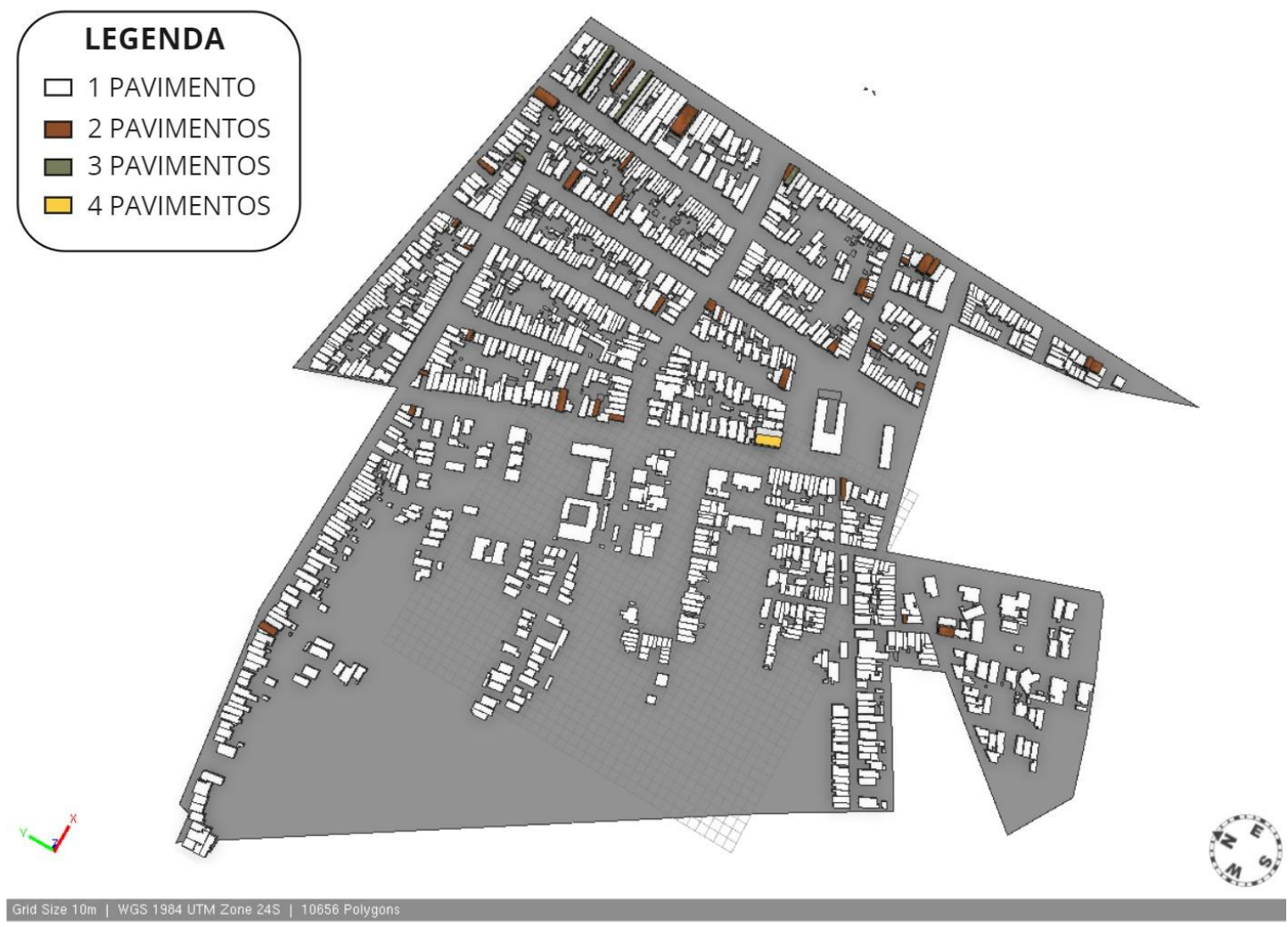

Fonte: Autores (2021)

Como produto final da aplicação do algoritmo, com as regras já mencionadas, obteve-se a modelagem 3D do bairro, sendo modeladas 1.496 edificações, geradas em poucos segundos após a sua aplicação, o que demonstra uma enorme eficácia do programa na geração de modelagem em grandes escalas, facilitando a etapa de modelagem, que por outros métodos é algo bem demorado e depende de computadores potentes e com grande processador. Imagens da modelagem podem ser vistas nas Figuras 13 e 14 .

Figura 13: Visualização tridimensional de dados GIS.
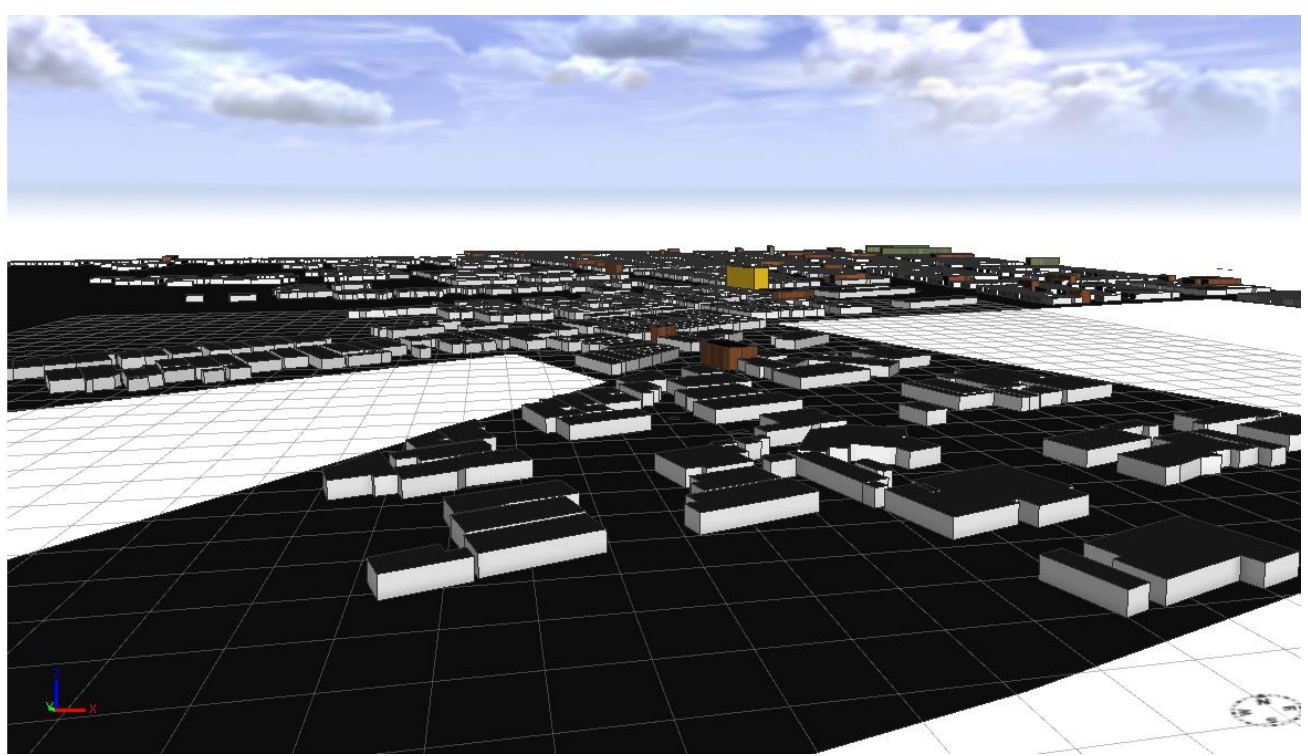

Fonte: Autores (2021) 
Research, Society and Development, v. 11, n. 2, e46711225930, 2022

(CC BY 4.0) | ISSN 2525-3409 | DOI: http://dx.doi.org/10.33448/rsd-v11i2.25930

Figura 14: Visualização tridimensional do bairro.

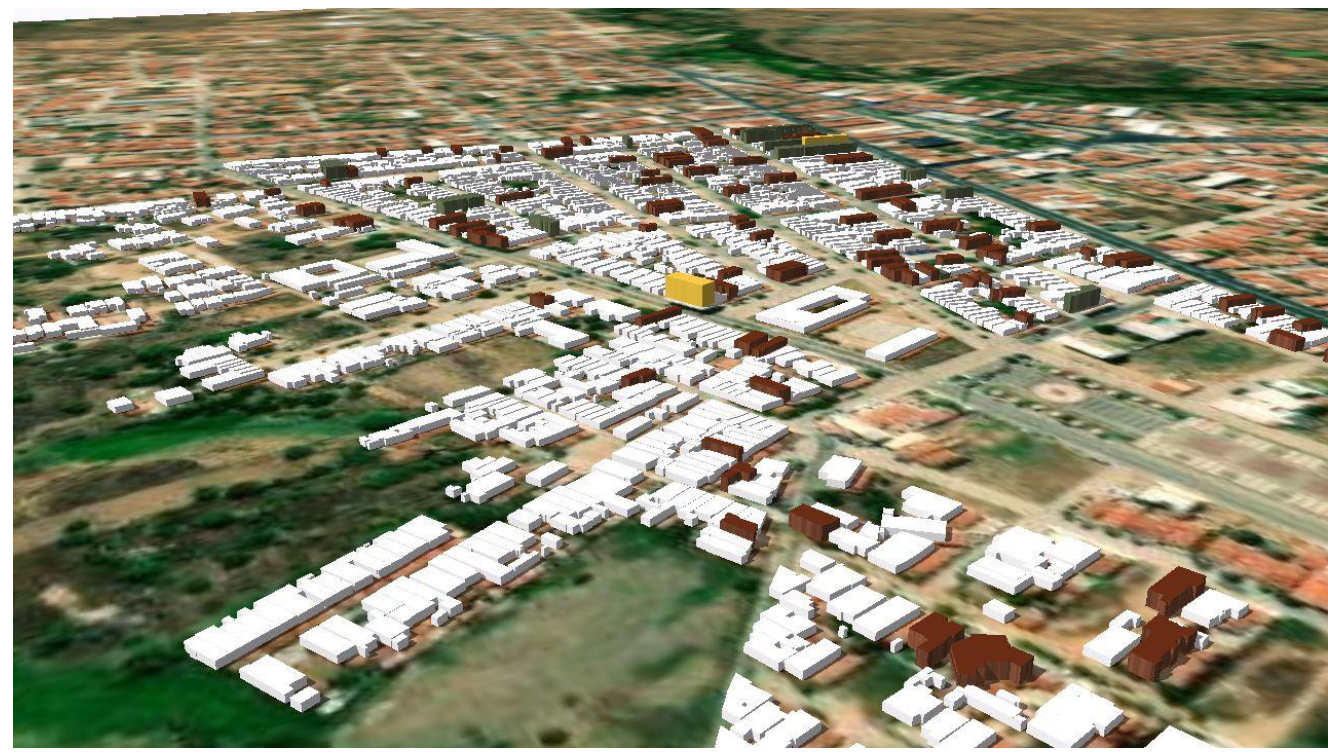

Fonte: Autores (2021).

Por meio do mapeamento elaborado através da modelagem é possível notar a forma de ocupação espontânea e sem ordenamento, ou seja, a análise favorece o entendimento do espaço urbano do bairro e a visualização de sua forma. Outro aspecto relevante obtido com a modelagem é a extração de informações de forma automática, como por exemplo, a área construída, área de projeção e gabarito, como pode ser observado na Figura 15.

Figura 15: Informações geradas.
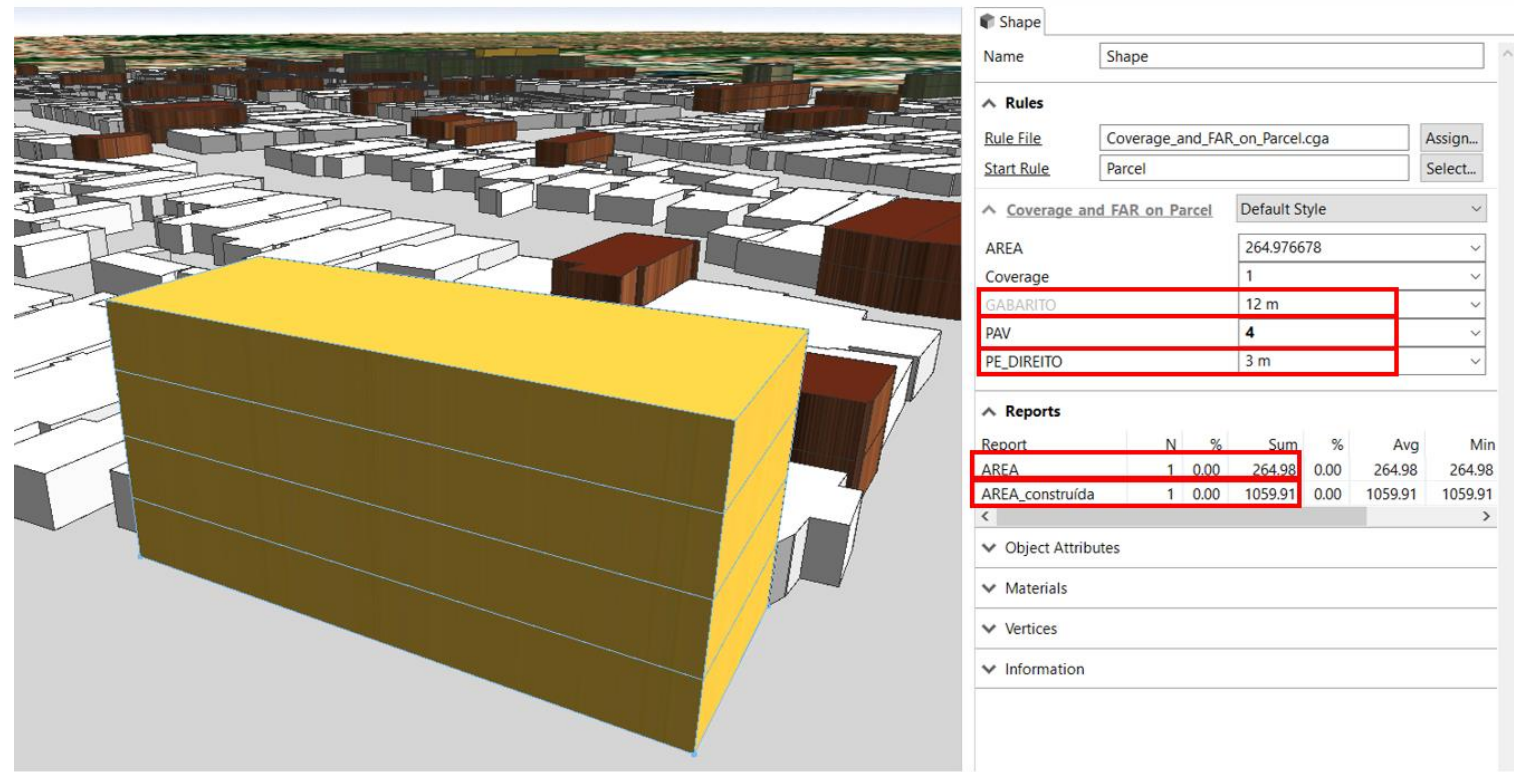

Fonte: Autores (2021)

Com os resultados obtidos, pode-se destacar os principais pontos positivos que foram viabilizados pela abordagem da metodologia GIS-BIM, tornando a modelagem rica em informações, e com um ganho de tempo extremamente relevante. Além de identificar a possibilidade de adaptação dessa metodologia, para que a mesma possa ser aplicada em diferentes situações, seja para avaliação de índices urbanísticos, conferências de compatibilidade com plano diretor, entre outras. 


\section{Considerações Finais}

O presente estudo abordou a integração GIS-BIM, por meio da elaboração de diretrizes com a utilização da metodologia processual, e através da sua aplicação é possível obter um conjunto de soluções, que se adaptem conforme a necessidade e prioridade desejada na modelagem levando em consideração parâmetros de gabarito, área construída e área de projeção da edificação. Com sua aplicação, demonstrou-se que esse método, facilita a etapa de modelagem em grandes escalas, tanto em termos de tempo desprendido, como na obtenção de informações por meio dessa modelagem, e o principal, que é possível tornar essa modelagem acessível aos planejadores ou responsáveis por tomadas de decisões urbanas.

Como principal dificuldade desse estudo, pode-se destacar o manuseio dos softwares, visto que são encontradas várias pesquisas com o uso dos mesmos, mas poucas que tratem sobre o uso de forma descritiva. O CityEngine é um software estrangeiro e de uso escasso no Brasil, o que dificulta ainda mais o processo de elaboração de algoritmos e regras paramétricas.

Pode-se denotar como contribuição desta pesquisa a elaboração de diretrizes capazes de integrar duas ferramentas de grande visibilidade como GIS e BIM, com um intuito de facilitar a modelagem urbana. Sendo assim um elemento facilitador de análise e monitoramento do planejamento urbano, demonstrando-se como um método de vasta aplicabilidade nos diversos setores de uma cidade, onde fica aberta diversas opções de pesquisas futuras, desde sua aplicação com a caracterização de outros componentes do planejamento urbano como a identificação do macrozoneamento, a identificação dos tipos de usos das edificações, e também com a aplicação de estudos voltados para as redes urbanas, tanto de transporte, como de abastecimento de água e energia.

Diante do exposto, pode-se concluir que, embora apresente limitações, sobretudo devido aos desafios que a ferramenta impõe, o presente estudo serviu como base, para que pesquisas futuras venham a incrementar mais regras e funções ao algoritmo, de modo a extrair ainda mais informações de forma prática, rápida e segura. O estudo indicou o GIS-BIM como uma abordagem mais flexível, que pode lidar com a complexidade e as mudanças que caracterizam as sociedades urbanas contemporâneas, mostrando se tratar de uma ferramenta útil nos processos de tomada de decisão na gestão urbana.

\section{Referências}

Arrubla, A. P., \& Chávez, J. V. P. (2016). Modelo de desarrollo local para una zona de Bogotá-Colômbia, relacionando a estrutura ecológica principal e o processo de expansão urbana. Revista UDCA Actualidad \& Divulgación Científica, 19 (2), 445-455. http://www.scielo.org.co/scielo.php?script=sci_arttext\&pid=S0123-42262016000200021\&lng=en\&tlng=es.

Almeida, F. A. da S., Andrade, M. L. V. X. (2018). Considerações sobre o conceito de City Information Modelagem. inSitu.

Barbosa, G. (2013). O discurso da sustentabilidade expresso no projeto urbano. Universidade Federal do Rio de Janeiro.

Costa, O. C., Reis Neto, J. F., \& Oliveira, A. P. G. (2020). Soil use and occupation in Caracol Settlement: a multitemporal assessment using remote sensing and geoprocessing techniques. Research, Society And Development, 9(12). http://dx.doi.org/10.33448/rsd-v9i12.11029

Costa Júnior, H. \& Cabral, S. C. (2019). Tecnologias no mapeamento de áreas de risco de deslizamento em Teófilo Otoni. Research, Society And Development, 8(12). https://doi.org/10.33448/rsd-v8i12.1353

Dantas, J. A. da C. (2013) Parâmetros urbanísticos na regulação do uso e ocupação do solo: estudo da densidade e do coeficiente de aproveitamento nos planos diretores de natal de 1994 e 2007. Universidade Federal do Rio Grande do Norte, Natal.

Deritti, M. D. (2018). O surgimento do conceito CIM através da interoperabilidade entre BIM e GIS e sua importância para as SMARTS CITIES. Revista Técnico-Científica do Crea-PR, Paraná.

Dresch, A., Lacerda, D. P., \& Antunes Júnior, J. A. V. (2015). Design Science Research: método de pesquisa para avanço da ciência e tecnologia. Bookman Editora.

Fink, A. (2005). Conducting research literature reviews: From the Internet to paper. Thousand Oaks: Sage.

IBGE. Censo demográfico 2010: resultados gerais da amostra. (2012).

Instituto Polis, Câmara dos Deputados/Comissão De Desenvolvimento Urbano e Interior. (2002). Estatuto da Cidade: guia para implementação pelos municípios e cidadãos. Brasília. 
Research, Society and Development, v. 11, n. 2, e46711225930, 2022

(CC BY 4.0) | ISSN 2525-3409 | DOI: http://dx.doi.org/10.33448/rsd-v11i2.25930

Khemlani, L. (2005). Hurricanes and their aftermath: how can technology help? AECbytes. https://goo.gl/vdcCUn

Lacerda, D. P., Dresch, A., Proença, A., Antunes Júnior, J. A. V. (2013). Design Science Research: método de pesquisa para a engenharia de produção. Gestão \& Produção. 20(4)

Liu, X. et al. (2017). A state-of-the-art review on the integration of Building Information Modeling (BIM) and Geographic Information System (GIS). International Journal of Geo- Information, 6(2).

Lukka, K. (2003). The Constructive Research Aproach. Publication of the Turku School of Economics and Business Administration. 83-101.

March, S. T., Smith, G. F. (1995). Design and natural science research in Information Technology. Decision Suport Systems, 15, 251-266. https://www.sciencedirect.com/science/article/abs/pii/0167923694000412.

Monteiro, A. R., \& Veras, A. T. de R. (2017). A questão habitacional no Brasil. Mercator - Revista de Geografia da UFC, 16(7), 1-12.

Projeto Smart City, Núcleo de pesquisa e extensão Acesso à Terra Urbanizada. (2020). Universidade Federal Rural do Semi-àrido, Brasil.

Osipov, M P; Chekodaev, O A. (2019). Optimization of the process of 3D visualization of the model of urban environment objects generated on the basis of the attributive information from a digital map. V International Conference On "Information Technology And Nanotechnology", [S.I], (2416), 535-541.

Olszewski, R., Turek, A., Laczynski, M. (2016). Urban Gamification as a Source of Information for Spatial Data Analysis and Predictive Participatory Modelling of a City's Development. In Proceedings of the 5th International Conference on Data Management Technologies and Applications. 176-181.

Reitz, T., Banz, S. S. (2014). The Esri 3D City Information Model. Proceedings 8th International Symposium of the Digital Earth. Malásia.

Sousa, R. de L., Vale, J. B., Barbosa, A. J. S. da S., Silva, T. L. \& Sousa, V. C. (2021). Diagnóstico do uso e cobertura de terra e a qualidade da água superficial da micro bacia do Pau Amarelo em São Francisco do Pará. Research, Society And Development, 10(5).

Wieringa, R. (2009). Design science as nested problem solving. Proceedings of the 4th int. conf. on design science research in information systems and technology. 